\title{
Eficácia de um programa de estimulação de capacidades intelectuais ${ }^{1}$
}

\author{
Efficacy of a program for stimulation \\ of intellectual capacities
}

\author{
Marília ZAMPIERI \\ Patrícia Waltz SCHELINI ${ }^{3}$ \\ Carolina Rosa CRESPO 3
}

\begin{abstract}
Resumo
O modelo Catell-Horn-Carroll divide a inteligência em dez fatores gerais, decompostos em fatores específicos. Estudos da área abordam a possibilidade de estimulação da inteligência, por meio de programas específicos para esse fim. O objetivo do presente estudo foi desenvolver atividades para estimulação de três componentes desse modelo - Inteligência Fluida, Inteligência Cristalizada e Memória de Curto Prazo -, bem como implementar um programa de estimulação e avaliar sua eficácia. 0 programa foi realizado ao longo de 13 sessões. Participaram do estudo 22 crianças com idade entre 7 e 10 anos, divididas em dois grupos para a realização das atividades. Os instrumentos utilizados no pré e pós-teste foram a Escala Weschsler de Inteligência para Crianças - III, o Teste de Desempenho Escolar e as Matrizes Progressivas Coloridas de Raven. Os resultados apontaram aumento significativo no escore total da Escala Wechsler em todas as análises e no Teste de Desempenho Escolar, no que se refere à estimulação da inteligência cristalizada e memória de curto prazo, o que parece ser indicativo da eficácia do programa.
\end{abstract}

Unitermos: Cognição. Inteligência. Técnicas cognitivas.

\begin{abstract}
Cattell-Horn-Carroll Model splits intelligence into ten general factors, broken down into specific factors. Studies in the area have discussed whether or not cognitive stimulation is possible through programs designed to do so. The aims of the present study were to develop tasks in order to stimulate three factors of Cattell-Horn-Carroll model-Fluid Intelligence, Crystallized Intelligence and Short-term Memory, to present a program comprised of these tasks, and to assess its efficacy. Program was developed during the course of thirteen sessions. Participants were 22 schoolchildren, age-range from 7 to 10 years, divided into two groups to perform activities. They were evaluated by Wechsler Intelligence Scalefor Children III, School Performance Test and Raven's Colored Progressive Matrices during pre and post-test. Results showed significant rise in total score for Wechsler Intelligence Scale for Children III in all analyses; and in School Performance Test for crystallized intelligence and short-term memory, which seems to be indicative of program efficacy.
\end{abstract}

Uniterms: Cognitive. Intelligence. Cognitive thecniques.

1 Apoio: Pró-Reitoria de Extensão da Universidade Federal de São Carlos.

2 Universidade Federal de São Carlos, Centro de Ciências Humanas, Programa de Pós-Graduação em Psicologia. Rod. Washington Luís, km 235, SP-310, 13565-905, São Carlos, SP, Brasil. Correspondência para/Correspondence to: M. ZAMPIERI. E-mail: <mazampieri@hotmail.com>.

3 Universidade Federal de São Carlos, Departamento de Psicologia. São Carlos, SP, Brasil. 
A inteligência parece ser um construto psicológico relacionado a muitos fenômenos sociais, dentre os quais o rendimento acadêmico, o rendimento no trabalho, a vulnerabilidade aos acidentes e a longevidade (Colom, 2006). Por isso, o interesse sobre o assunto aumenta à medida que alterações nas capacidades intelectuais podem influenciar outros fatores. Justamente por sua relevância, a Psicologia explora a inteligência humana há mais de 100 anos. No entanto, não existe uma definição universalmente aceita para esse construto, como tantos outros inerentes à ciência psicológica (J.M. Oliveira-Castro \& K.M. Oliveira-Castro, 2001; Primi, 2002).

Uma das principais correntes no estudo da inteligência é a psicométrica, que busca compreendê-la em termos de estruturas chamadas "fatores". No final da década de 1990, McGrew e Flanagan apresentaram o modelo Cattell-Horn-Carroll de inteligência, produto da conciliação de teorias apresentadas anteriormente por Cattell (1971; 1987), Horn (1985; 1991) e Carroll (1993). O Modelo das Capacidades Cognitivas de Cattell-Horn-Carroll (CHC), como representante da corrente psicométrica, propõe a existência de dez capacidades gerais: Inteligência Fluida (Gf), Conhecimento Quantitativo (Gq), Inteligência Cristalizada (Gc), Memória de Curto Prazo (Gsm), Processamento Visual (GV), Processamento Auditivo (Ga), Armazenamento e Recuperação Associativa a Longo Prazo (G/r), Velocidade de Processamento Cognitivo (Gs), Tempo/Velocidade de Decisão/Reação (Gt) e Leitura-Escrita (Grw). As capacidades gerais são representadas por 73 capacidades específicas (Schelini \& Wechsler, 2005; Schelini, 2006). Todavia, apenas capacidades gerais de Inteligência Fluida, Inteligência Cristalizada e Memória de Curto Prazo serão aqui descritas, por serem aquelas que recebem destaque no presente estudo.

A Inteligência Fluida é uma habilidade de raciocínio associada a componentes não verbais, pouco dependente de conhecimentos adquiridos previamente e de influências culturais, parecendo receber influência de alterações orgânicas (Alfonso, Flanagan \& Radwan, 2005; Schelini, 2006). As operações mentais que as pessoas utilizam frente a uma tarefa relativamente nova e que não podem ser executadas automaticamente representam Gf (Horn, 1991), por isso pode ser definida como

354 a flexibilidade e capacidade de análise e adaptação dian- te de situações-problema não experienciadas previamente (Cole \& Randall, 2003). Portanto, envolve o reconhecimento e formação de conceitos, a compreensão de implicações, a resolução de problemas e a reorganização ou transformação de informações (Schelini, 2002).

Já a Inteligência Cristalizada é o conjunto de capacidades exigidas na resolução de problemas cotidianos, sendo dependente de conhecimentos adquiridos pela experiência educacional e contato com a cultura (Alfonso et al., 2005; Schelini, 2006). Em função disso, supõe-se que Gc possa sempre ser incrementada, à medida que se acumulam novas experiências com o passar da idade, ao contrário do que é proposto para Gf, mais limitada pelo desenvolvimento biológico.

Por fim, a Memória de Curto Prazo consiste na apreensão e uso das informações por um período curto de tempo. Passado esse tempo e uma vez utilizada a informação, ela poderá ser descartada caso não seja transferida para a memória de longo prazo. A capacidade da Gsm é limitada a cerca de oito estímulos ou informações, pois ela se refere à informação que está sendo utilizada para realizar as tarefas (Colom \& Flores-Mendoza, 2006). Deficits no desempenho em tarefas que envolvem Gsm são caracterizados pela dificuldade em recordar uma informação recém-adquirida (Schelini, 2006).

A apresentação empiricamente avaliada de capacidades que compõem a inteligência, como a realizada pelo modelo CHC, facilita o delineamento daquilo que deverá ser avaliado e, consequentemente, proporciona uma compreensão mais precisa da estrutura da inteligência, ocasionando maior facilidade para a elaboração de estratégias de intervenção. No que se refere à intervenção ou às estratégias de estimulação das capacidades cognitivas, vale ressaltar, primeiramente, que vários estudos indicam que tais capacidades são maleáveis ou passíveis de modificação por meio de diferentes tipos de ação (Bransford \& Stein, 1993; Detterman \& Sternberg, 1982; Grotzer \& Perkins, 2000; Mayer, 2000; Perkins \& Grotzer, 1997). Neste momento, vale esclarecer que há estudos voltados a tentativas de desenvolvimento das capacidades intelectuais e também há de se citar a ocorrência de um aumento "natural" dos escores em medidas de inteligência ao longo do tempo. Esse ganho nas medidas intelectuais é conhecido como 
Efeito Flynn, nome devido ao psicólogo americano James R. Flynn, que primeiro o documentou. De forma geral, os ganhos em termos de Ql são de aproximadamente três pontos por década (ou 7,5 pontos por geração), entre crianças no período escolar, e de cinco pontos entre adultos (Flynn, 2006; Lynn \& Harvey, 2008).

Dentre as ações já efetuadas como forma de estimular as capacidades intelectuais, é possível citar o Projeto Milwaukee (Colom, 2002), o programa Early Head Start (Ayoub et al., 2009) e o Projeto Abecedário (Campbell \& Ramey, 1994). O primeiro projeto consistiu no ensino de mães para estimular diariamente seus filhos desde o nascimento até os seis meses de vida; o projeto Abecedário também era composto por sessões diárias de estimulação precoce, além de acompanhamento médico e nutricional. Já o projeto Early Head Start é um programa do governo federal dos Estados Unidos que tem como objetivo promover suporte a famílias de baixa renda. Parte do projeto consiste na orientação dos pais para a promoção de adequadas condições ambientais que visem favorecer o desenvolvimento cognitivo das crianças. Esses programas obtiveram resultados satisfatórios com a estimulação de crianças em contato com fatores de risco para o desenvolvimento cognitivo, como condição socioeconômica desfavorável e propensão a retardo mental. Dentre os ganhos obtidos, pode-se citar aumento nos escores apresentados nos testes de inteligência e maior tendência a ingresso em cursos superiores (Campbell \& Ramey, 1994; Colom, 2002). Além disso, foi possível identificar que a presença de fatores de risco, como baixa escolaridade das mães e baixa estimulação de capacidades cognitivas, incluindo a linguagem, apresentou-se associada a índices mais baixos de desenvolvimento cognitivo (Ayoub et al., 2009).

Em Portugal, Almeida e Morais (1997; 2002) implementaram versões do Programa de Promoção Cognitiva. As atividades do programa foram elaboradas tendo como referencial a concepção de inteligência como um conjunto de capacidades específicas que podem ser treinadas. Os autores defendem que o treino cognitivo possibilitaria um "aprender a pensar"e, consequentemente, um "aprender a aprender", e ressaltam a importância de que as atividades sejam extrapoladas para o dia a dia dos participantes. Além de garantir maior motivação, essa estratégia aumenta a possibili- dade de consolidação, transferência e aplicação do processo a diversas situações. O programa foi realizado ao longo de 15 sessões em grupo, divididas em quatro módulos: um introdutório e os demais referentes aos três estágios de processamento da informação: recepção e organização da informação, relacionamento da informação e elaboração das respostas. O programa existe desde o final da década de 1980 e tem passado por reformulações e aprimoramentos desde então. Os resultados têm indicado, de acordo com os autores, a eficácia do programa quanto ao desempenho em tarefas que envolvem resolução de problemas, por meio de treino sistemático e intencional (Almeida \& Morais, 2002).

Para a constituição de um projeto de estimulação cognitiva, é necessário definir quais capacidades relativas à inteligência deseja-se promover, para que, então, atividades possam ser elaboradas, a fim de atingir os objetivos do programa (Almeida \& Morais, 2002). A estimulação cognitiva pode ser feita por meio de atividades simples, cotidianas e lúdicas, utilizando jogos tradicionais que, por exemplo, exercitem a atenção, a memória e a relação causa-efeito, e que favoreçam o planejamento, pois o resultado do jogo depende das ações do jogador. A realização em grupo dessas atividades favorece a discussão sobre diferentes estratégias levantadas para a resolução dos problemas apresentados (Muragaki, Okamoto, Furlan \& Toldrá, 2006).

Existem outros fatores importantes para o sucesso dos programas de estimulação cognitiva: esclarecer os objetivos das atividades e destacar sua importância para os participantes, como forma de garantir sua motivação e engajamento (Almeida \& Morais, 2002); estar o aplicador do programa atento às necessidades e desempenho de cada participante, para garantir que a estimulação se aproxime o máximo possível das demandas individuais. Além disso, o aplicador do programa atua como mediador da aprendizagem, sendo responsável por conduzir os participantes a ficarem sob controle dos aspectos relevantes das tarefas apresentadas, facilitando o processo de aprendizagem (Gomes, 2002).

Atividades para estimulação cognitiva podem ser realizadas em contextos educacionais, hospitalares e extrainstitucionais. Elas podem ter caráter preventivo, quando aplicadas a populações de risco, ou caráter de reabilitação, no caso de danos neurológicos produzidos 
por diversas condições. Diante disso, é possível perceber que os ganhos produzidos pela estimulação cognitiva vão além de aumentar o desempenho escolar: englobam aumento da autoestima, satisfação no trabalho, sucesso na resolução de problemas cotidianos, à medida que o indivíduo passa a estabelecer uma relação entre seu próprio comportamento e os resultados produzidos (Almeida \& Morais, 1997).

Trabalhos nacionais que tiveram como objetivo a estimulação cognitiva não são muito numerosos até o presente. Alguns dos estudos encontrados referem-se ao treino de habilidades cognitivas em idosos (Chariglione, 2010; Irigaray, 2009), justificado pelo risco às funções cognitivas decorrente de doenças neurodegenerativas, como o Mal de Alzheimer. Em relação à população em idade escolar, o estudo de Aquino (1999) ilustra a investigação da adequação do Programa de Promoção Cognitiva (Almeida \& Morais, 1997; 2002) à população brasileira. Porém, ainda há carência de estudos nacionais que representem a tentativa de elaborar um programa de estimulação voltado às capacidades do modelo CHC e que, ao mesmo tempo, investiguem a eficácia de sua implementação. Diante disso, os objetivos do presente estudo envolveram a elaboração e implementação de atividades de estimulação dos fatores Gf, Gc e Gsm do modelo CHC, bem como a investigação da eficácia das atividades em relação à estimulação dos três fatores.

\section{Método}

\section{Participantes}

Participaram do estudo 22 crianças, com idade entre 7 e 11 anos (média de idade = 8,89 anos, desvio-padrão = 1,19), de ambos os gêneros, sendo 8 do gênero feminino e 14 do masculino, que estudavam em uma escola estadual de um município do interior de São Paulo. Os participantes foram selecionados por frequentarem um programa de ensino de leitura para estudantes com dificuldades de leitura e escrita. Foram formados dois grupos para a realização do procedimento, um com 15 participantes e outro com 7, conforme o horário que o aluno frequentava aquele programa, o que explica a diferença numérica entre os grupos. O estudo foi aprovado pelo Comitê de Ética em Pesquisa da Universi- dade Federal de São Carlos, sob Protocolo n 010/2007, em 29 de janeiro de 2007, e os responsáveis por todos os participantes assinaram um Termo de Consentimento Livre e Esclarecido antes de sua inclusão na amostra.

\section{Instrumentos}

Foram utilizados três instrumentos: Teste de Desempenho Escolar (TDE) (Stein, 1994); a Escala Wechsler de Inteligência para Crianças - Terceira Edição (WISC III) (Wechsler, 2002), para avaliar a inteligência cristalizada e a memória de curto prazo; e as Matrizes Progressivas Coloridas de Raven (Angelini, Alves, Custódio, W.F. Duarte, J.L.M. Duarte, 1999), para avaliar a inteligência fluida.

A Escala Wechsler de Inteligência para Crianças - Terceira Edição é destinada a crianças de 6 anos a adolescentes de 16 anos e 11 meses, sendo dividida em duas escalas, verbal e de execução. A escala verbal é composta pelos subtestes de informação, compreensão, aritmética, semelhanças, vocabulário e dígitos. A escala de execução é composta por sete subtestes: completar figuras, arranjo de figuras, cubos, armar objetos, código, procurar símbolos e labirintos. A aplicação do instrumento é individual e leva de 50 a 70 minutos. A escala permite a obtenção do QI total, QI verbal e QI de execução, além de escores referentes a quatro índices fatoriais: compreensão verbal, organização perceptual, resistência à distração e velocidade de processamento.

As Matrizes Progressivas Coloridas de Raven são compostas de três séries de 12 figuras, nas quais sempre há um pedaço em branco. Abaixo de cada figura, há seis opções de desenhos que completam a figura principal, devendo o participante apontar qual das opções completa corretamente a figura.

OTeste de Desempenho Escolar é composto de três seções: escrita, aritmética e leitura. Na primeira, o avaliado deve escrever palavras que lhe são ditadas; na parte de aritmética, deve resolver algumas operações matemáticas; e, na última seção, deve ler uma lista de 70 palavras.

Além desses instrumentos, alguns materiais foram utilizados durante a realização das atividades que compuseram o programa de estimulação, como lápis, borracha, folhas de atividades e tabuleiros de jogos, entre outros. 


\section{Procedimento}

O projeto foi submetido à aprovação do Comitê de Ética em Pesquisa da Universidade e, após obtenção de parecer favorável, foi feita seleção dos participantes e contato com os responsáveis para formalização do consentimento para participação no estudo. Feito isso, ocorreu a aplicação individual do TDE, WISC III e Matrizes Progressivas Coloridas de Raven, cujas pontuações compuseram os dados do pré-teste. A aplicação dos instrumentos ocorreu em quatro encontros: no primeiro deles foi aplicado o TDE; no segundo, as Matrizes Progressivas Coloridas de Raven; para a aplicação do WISC III foram necessários dois encontros (o terceiro e o quarto). Essa divisão foi feita para que a duração de cada encontro não se tornasse exaustiva para os participantes.

Em seguida, foram iniciadas as atividades do Programa de Estimulação. As quatro etapas (motivação, resolução de problemas, estimulação de capacidades específicas da inteligência e encerramento) ocorreram ao longo de treze sessões semanais, com duração média de 40 minutos. A primeira sessão, correspondente à etapa de motivação, foi destinada à apresentação dos integrantes do grupo, explicação dos objetivos do programa e elaboração conjunta das regras do grupo, a fim de promover a integração e engajamento dos participantes nas atividades subsequentes.

A etapa de resolução de problemas foi realizada em duas sessões, tendo como objetivo apresentar atividades cuja realização envolvesse a identificação do problema, o levantamento de estratégias para solucioná-lo e a escolha da estratégia que se apresentasse mais adequada.

A terceira etapa foi formada por três módulos, cada qual composto por atividades designadas à estimulação de uma das três capacidades contempladas pelo programa: Gsm, Gc e Gf. Cada módulo teve a duração de três sessões, totalizando nove sessões.

Durante o módulo de estimulação da Gsm, foram realizadas oito atividades. Durante todas elas, eram apresentadas algumas informações aos participantes (como um conjunto de figuras, preços de produtos, posição de pontos em um diagrama), que deveriam observá-las e memorizá-las. Depois de recolhido o material com essas informações, os participantes eram solicitados a apresentar as informações memorizadas, localizando as figuras memorizadas diante de um conjunto maior, ou ligando informações de duas colunas, entre outras tarefas.

O módulo de estimulação da Gc também foi composto por oito atividades. Uma delas, denominada Quem ou o que sou eu, consistia na leitura, pelo pesquisador, de adivinhações, ou seja, frases que continham informações sobre um objeto ou pessoa, que deveria ser nomeado (a) pelos participantes. Um exemplo desta atividade é a proposição Sou a cor do céu, da água da piscina e de alguns olhos, cuja resposta esperada era azul.

O último módulo dessa etapa consistiu na realização de cinco atividades para estimulação da Gf. A atividade Agrupando compôs este módulo, cujos participantes recebiam uma variedade de folhas de árvores e eram solicitados a agrupá-las de acordo com critérios estabelecidos por eles, como forma, tamanho e cor. Cada conjunto deveria ser composto por folhas com pelo menos uma característica em comum, sendo que tal característica não poderia estar presente em folhas alocadas nos demais conjuntos.

Após a realização de cada atividade, o pesquisador questionava os participantes sobre a estratégia empregada na resolução e também sobre a eficácia de tais estratégias. Essa discussão tinha como objetivo levar os participantes a relacionar as estratégias utilizadas com o desempenho na tarefa: tarefas realizadas com êxito eram indicativas do ajuste da estratégia à demanda.

A última sessão do programa foi destinada ao encerramento das atividades. Durante esta sessão foi confeccionado um cartão, assinado e desenhado também pelos demais participantes, como forma de construir uma lembrança do grupo. Após dois meses do término da última sessão do programa, foram reaplicados os instrumentos TDE, WISC III e Matrizes Progressivas Coloridas de Raven. As pontuações obtidas nesta avaliação compuseram os dados do pós-teste.

\section{Resultados}

Para uma análise mais precisa da eficácia da estimulação de cada uma das capacidades, foram considerados os dados de participantes que presenciaram pelo menos duas das três sessões que compuseram cada um dos módulos. Isso explica a diferença no núme- 
ro de participantes para a análise da eficácia do programa de estimulação sobre cada uma das capacidades do Modelo Cattell-Horn-Carroll envolvidas no estudo. A Tabela 1 apresenta os dados resultantes do pré e pós-teste, referente às avaliações com o TDE, as Matrizes Progressivas Coloridas de Raven e o WISC III. Deste último instrumento, foram utilizados o escore total e os subtestes dígitos, Semelhanças, Informação, Vocabulário e Compreensão. O número 1 ao lado do nome do teste indica os escores do pré-teste, enquanto o número 2 indica os escores do pós-teste.

Para investigar o impacto do programa de estimulação sobre o fator Gf, foram utilizados os escores brutos obtidos no TDE, Matrizes Progressivas Coloridas de Raven e escore total do WISC III. Do total de participantes, 10 crianças atingiram o critério de presença no módulo de estimulação da Gf, tendo seus dados computados para análise.

Para a observação do impacto do programa sobre o fator Gsm, foram utilizados os escores brutos obtidos no TDE, WISC III e no subteste Dígitos do WISC III. Nesse módulo, 13 participantes estiveram presentes em pelo menos duas sessões.

Em relação ao módulo de estimulação do fator Gc, foram utilizados os escores brutos do TDE e WISC III, além dos subtestes do WISC III Semelhanças, Informação, Vocabulário e Compreensão. Foram computados os dados de 13 participantes.

Para a maioria dos pares de dados, os desvios-padrão e erros-padrão apresentados pelos escores do pós-teste foram maiores. Os pares de dados que não apresentam tal característica são os referentes aos escores do Raven, no módulo Gf; escore total do WISC III e subtestes Vocabulário e Compreensão, no módulo Gc; e o escore total do WISC III e do subteste Dígitos, no módulo Gsm.

Para verificar se a diferença entre os escores obtidos no pré e pós-teste foi significativa, os dados foram submetidos à análise do teste $t$ para dados pareados. Os resultados são apresentados na Tabela 2.

Tabela 1. Pontuações no pré e pós-teste nos instrumentos de avaliação das capacidades específicas avaliadas. São Carlos (SP), 2007.

\begin{tabular}{|c|c|c|c|c|c|c|c|}
\hline Capacidades & Instrumento & Média & $n$ & Desvio-Padrão & Erro-Padrão & Mínimo & Máximo \\
\hline \multirow[t]{6}{*}{ Gf } & Raven 1 & 18,90 & 10 & 5,32 & 1,68 & 6 & 27 \\
\hline & Raven 2 & 21,30 & 10 & 4,42 & 1,39 & 10 & 29 \\
\hline & TDE 1 & 41,11 & 10 & 31,01 & 10,33 & 2 & 81 \\
\hline & TDE 2 & 58,33 & 10 & 38,09 & 12,69 & 4 & 111 \\
\hline & WISC 1 & 135,20 & 10 & 37,44 & 11,84 & 65 & 189 \\
\hline & WISC 2 & 158,00 & 10 & 40,83 & 12,91 & 91 & 240 \\
\hline \multirow[t]{12}{*}{ Gc } & WISC 1 & 131,53 & 13 & 31,63 & 8,77 & 75 & 174 \\
\hline & WISC 2 & 156,23 & 13 & 26,41 & 7,32 & 124 & 216 \\
\hline & TDE 1 & 37,00 & 13 & 30,83 & 8,55 & 1 & 81 \\
\hline & TDE 2 & 59,93 & 13 & 33,84 & 9,04 & 6 & 111 \\
\hline & Informação 1 - & 7,54 & 13 & 1,66 & 0,46 & 5 & 11 \\
\hline & Informação 2 & 8,77 & 13 & 2,52 & 0,69 & 5 & 13 \\
\hline & Vocabulário 1- & 14,15 & 13 & 6,15 & 1,70 & 3 & 23 \\
\hline & Vocabulário 2 & 14,69 & 13 & 3,77 & 1,04 & 7 & 21 \\
\hline & Semelhança 1- & 4,92 & 13 & 2,39 & 0,66 & 0 & 8 \\
\hline & Semelhança 2 & 8,61 & 13 & 7,19 & 1,99 & 4 & 32 \\
\hline & Compreensão 1- & 9,38 & 13 & 3,66 & 1,01 & 3 & 15 \\
\hline & Compreensão 2 & 11,77 & 13 & 2,80 & 0,77 & 6 & 17 \\
\hline \multirow[t]{6}{*}{ Gsm } & TDE 1 & 33,75 & 12 & 26,54 & 7,66 & 1 & 81 \\
\hline & TDE 2 & 53,50 & 12 & 38,22 & 11,03 & 4 & 111 \\
\hline & Dígitos 1 & 8,15 & 13 & 2,67 & 0,74 & 2 & 14 \\
\hline & Dígitos 2 & 8,92 & 13 & 1,98 & 0,54 & 5 & 12 \\
\hline & WISC 1 & 125,15 & 13 & 34,11 & 9,46 & 65 & 189 \\
\hline & WISC 2 & 148,85 & 13 & 31,79 & 8,81 & 91 & 240 \\
\hline
\end{tabular}

TDE: Teste de Desempenho Escolar; WISC: Escala Wechsler de Inteligência para Crianças; Gf: Inteligência Fluida; Gc: Inteligência Cristalizada; Gsm: 
Tabela 2. Teste $t$ para dados pareados. São Carlos (SP), 2007.

\begin{tabular}{|c|c|c|c|c|c|c|c|}
\hline Capacidades & Instrumento & Diferença entre as médias & Desvio-Padrão & Erro-Padrão & $t$ & Graus de liberdade & Significância \\
\hline \multirow[t]{5}{*}{ Gf } & Raven 1 - Raven 2 & $-2,40$ & 4,72 & 1,49 & $-1,606$ & 9 & 0,142 \\
\hline & TDE 1 - TDE 2 & $-17,22$ & 27,36 & 9,12 & $-1,889$ & 9 & 0,960 \\
\hline & WISC $1-$ WISC 2 & $-22,80$ & 14,82 & 4,68 & $-4,864$ & 9 & $0,001^{* *}$ \\
\hline & TDE 1-TDE 2 & $-21,54$ & 19,79 & 5,49 & $-3,923$ & 12 & $0,002^{*}$ \\
\hline & $\begin{array}{l}\text { WISC } 1 \text { - WISC } 2 \\
\text { Informação } 1\end{array}$ & $-24,63$ & 17,44 & 4,84 & $-5,103$ & 12 & $0,000^{* *}$ \\
\hline \multirow[t]{5}{*}{ Gc } & Informação 2 & $-1,23$ & 2,35 & 0,65 & $-1,888$ & 12 & 0,083 \\
\hline & $\begin{array}{l}\text { Vocabulário } 1 \\
\text { Vocabulário } 2\end{array}$ & $-0,54$ & 3,38 & 0,94 & $-0,0574$ & 12 & 0,577 \\
\hline & $\begin{array}{l}\text { Semelhanças 1- } \\
\text { Semelhanças } 2\end{array}$ & $-3,69$ & 7,79 & 2,16 & $-1,708$ & 12 & 0,113 \\
\hline & $\begin{array}{l}\text { Compreensão } 1 \\
\text { Compreensão } 2\end{array}$ & $2-, 38$ & 2,53 & 0,70 &,- 9161 & 12 & $0,005^{* *}$ \\
\hline & TDE1 - TDE2 & $-19,75$ & 24,07 & 6,95 & $-2,842$ & 11 & 0,0168 \\
\hline \multirow[t]{2}{*}{ Gsm } & Dígitos1 - Dígitos2 & $-0,77$ & 1,88 & 0,52 & $-1,477$ & 12 & 0,165 \\
\hline & WISC1 - WISC2 & $-23,69$ & 15,74 & 4,36 & $-5,427$ & 12 & $0,000^{* *}$ \\
\hline
\end{tabular}

${ }^{*}$ valores significativos para $p<0,05 ;{ }^{* *}$ valores significativos para $p<0,01$; TDE: Teste de Desempenho Escolar; WISC: Escala Wechsler de Inteligência para Crianças; Gf: Inteligência Fluida; Gc: Inteligência Cristalizada; Gsm: Memória de Curto Prazo; Raven: Matrizes Progressivas Coloridas de Raven.

Em relação ao módulo de estimulação da Gf, ou ao desempenho do total de participantes desse módulo, observam-se na Tabela 2 escores mais elevados no pós-teste. O teste no qual as médias de pontuação apresentaram uma maior diferença entre o início e o fim do projeto foi o WISC III, considerando-se o escore total ou somatória de pontos obtidos em todos os subtestes. No escore total do WISC III, houve um aumento significativo na média de pontuação $(p \leq 0,01)$. Já os resultados referentes ao Raven e ao TDE não apresentaram diferença significativa entre pré e pós-teste.

Em relação ao módulo de estimulação da $G c$, observa-se no desempenho dos participantes uma diferença significativa entre os dados das duas avaliações para o escore total do WISC III, do subteste Compreensão $(p \leq 0,01)$ eTDE $(p \leq 0,05)$. Nos demais subtestes do WISC III também é possível observar escores mais altos no pós-teste, embora não tenham apresentado diferença significativa.

O último conjunto de dados refere-se ao módulo de estimulação do fator Gsm. Comparando-se os escores totais do WISC III, encontra-se diferença significativa $(p \leq 0,01)$, assim como para o escore do TDE $(p \leq 0,05)$. Porém, para o subteste Dígitos, relacionado especificamente ao fator estimulado (Memória de Curto Prazo), a diferença entre pré e pós-teste não foi significativa.

\section{Discussão}

Diante da observação de escores mais altos no pós-teste para todos os escores analisados, é possível traçar algumas considerações sobre a eficácia do programa de estimulação cognitiva. Para os três conjuntos de dados, o escore total do WISC III obtido no pós-teste foi superior ao escore obtido no pré-teste. Pode-se argumentar que, no tempo decorrido entre as duas avaliações, o desenvolvimento e o aprendizado convencional - por meio da escola e estimulação via interação com pares e adultos - podem explicar o aumento nos escores obtidos. Porém, de acordo com Melo (2007), não se observam diferenças significativas na pontuação do WISC III dentro do período de um ano (para idades de 7, 8 e 9 anos) e de até dois anos (para faixas etárias mais elevadas, dos 10 aos 15 anos) $(p \leq 0,01)$. Segundo a autora, existe um aumento progressivo nas médias com o aumento da idade, mas esse aumento não é suficiente para resultar em diferenças significativas dentro das faixas etárias. O próprio WISC III (na sua adaptação para - Brasil) agrupa a maioria das faixas etárias em dois anos (7; 8-9; 10-11;12-13; 14-16). Sendo assim, pode-se considerar que a melhora nos escores dos participantes tenha sido produto da estimulação realizada durante o programa, em interação com outros fatores.

OTeste de Desempenho Escolar avalia o desempenho escolar em tarefas de leitura, escrita e aritmética. 
Foi incluído no conjunto de instrumentos de avaliação dos participantes devido ao fato de que os produtos da estimulação cognitiva podem não apenas se manifestar nos instrumentos de avaliação da inteligência, mas também ter implicações práticas, refletindo no desempenho dos indivíduos em tarefas escolares. Pode-se discutir desempenho significativamente maior para os participantes incluídos na análise da estimulação de Gc e Gsm, em partes, como ganhos provenientes da estimulação proporcionada pelo programa.

O instrumento Matrizes Progressivas Coloridas de Raven tem sido relacionado ao fator Gf (Lynn, 2009). Embora não tenha sido observada diferença significativa entre os desempenhos no pré e pós-teste no Raven, pode-se buscar o efeito da estimulação da inteligência fluida na realização de tarefas não diretamente desenvolvidas para sua estimulação ou avaliação, já que foi demonstrado que a Gf influencia a execução de tarefas que são descritas como relativas a outras capacidades (Rabbitt \& Stuart-Hamilton, 1997). Sendo assim, as atividades relacionadas ao desenvolvimento dessa capacidade específica Gf podem ter contribuído também para o aumento nos escores do WISC III, já que Gfé, dentre os fatores gerais do Modelo Cattell-Horn-Carroll, o que mais se associa à concepção do fator g, que, por sua vez, seria responsável pelos processos cognitivos comuns aos diferentes tipos de atividades mentais (Primi, 2002).

Os subtestes do WISC III Informação, Semelhanças, Compreensão e Vocabulário foram utilizados na análise da estimulação da Gc, conforme já descrito. Estes subtestes avaliam a quantidade de informação aprendida pelos indivíduos em sua experiência - característica definidora do fator Gc - e sua capacidade de verbalizar esse conteúdo aprendido. Embora não tenha sido constatada diferença significativa em todos os subtestes, pode-se considerar que as atividades desenvolvidas para estimulação desse fator tenham contribuído para promover o contato dos participantes com informações possivelmente não disponibilizadas em outros contextos, ou o exercício dessa capacidade, no caso de não proporcionar contato com informações novas.

Em relação à estimulação do fator Gsm, a diferença no escore do subteste dígitos também não foi significativa. Este era o único subteste diretamente relacionado ao fator em questão, devido ao número redu- zido de instrumentos nacionais que avaliem a memória de curto prazo. Apesar disso, são válidas as mesmas considerações feitas acerca do papel da inteligência fluida no desempenho de tarefas de natureza diversa. Segundo o modelo de processamento da informação baseado na teoria Gf-Gc e proposto porWoodcock (1998), entende-se a memória como uma capacidade elementar no funcionamento cognitivo, de modo que ela está envolvida em todo tipo de tarefa realizada. Assim, é possível que os ganhos proporcionados pelo programa de estimulação da memória de curto prazo sejam refletidos no desempenho em testes mais gerais, como oTDE e o WISC III, ainda que não sejam significativos no subteste especificamente designado a avaliar a memória de curto prazo.

A organização das sessões foi proposta para otimizar os ganhos promovidos pelo programa, de modo que as contribuições feitas ao fator Gsm pudessem ser exercitadas já nos módulos seguintes, de estimulação da inteligência cristalizada e da fluida. O módulo inicial de resolução de problemas também foi conduzido antes da estimulação de fatores específicos da inteligência, para que as tarefas de estimulação da Gsm, Gc e Gfrepresentassem oportunidades para o exercício do raciocínio desenvolvido nesse módulo inicial.

Pode-se considerar que o presente projeto tenha apresentado tarefas próximas do cotidiano dos participantes, conforme destacado por Almeida e Morais (1997), por exigirem o emprego de estratégias utilizadas no dia a dia. Novos projetos que continuem a enfocar essa questão e que envolvam um maior número de participantes mostram-se necessários para garantir o interesse e generalização do aprendizado.

Além disso, o programa pode ter atuado como fator de proteção, dado que a amostra era composta por participantes de uma população de risco, mais suscetível ao fracasso escolar. Ao incentivar o uso das estratégias de resolução de problemas, elas podem ser generalizadas para situações cotidianas e acadêmicas.

\section{Considerações Finais}

Em termos gerais, é possível perceber que o presente estudo alcançou parte dos objetivos. As atividades foram elaboradas com base em um referencial teórico, e muitas delas despertaram o interesse dos participantes, 
desafiando-os a superar as dificuldades e a empregar estratégias úteis na resolução dos problemas, ainda que o impacto do conjunto de sessões do programa não tenha se manifestado nos escores de todos os instrumentos utilizados na avaliação.

Estudos subsequentes podem contribuir para a elaboração e implementação de programas que variem alguns elementos em relação ao programa apresentado, como número de participantes por grupo de estimulação (a fim de compor uma amostra mais numerosa), capacidades específicas do modelo CHC a serem estimuladas, número de sessões, entre outros. A realização de programas nacionais de estimulação cognitiva ainda é muito escassa, e todo trabalho desenvolvido nessa área será de grande contribuição para a geração de conhecimento e promoção do desenvolvimento dos participantes envolvidos.

\section{Referências}

Alfonso, V. C., Flanagan, D. P., \& Radwan, S. (2005). The impact of the Cattell-Horn-Carroll theory on test development and interpretation of cognitive and academic abilities. In D. P. Flanagan \& P. L. Harrison. Contemporary intellectual assessment (2a ed., pp.185-202). Nova lorque: Guilford.

Almeida, L., \& Morais, M. F. (1997). Programa de promoção cognitiva (4a ed.). Barcelos: Didálvi.

Almeida, L., \& Morais, M. F. (2002). Programa de promoção cognitiva (4a ed.). Braga: Psiquilíbrios.

Angelini, A. L., Alves, I. C. B., Custódio, E. M., Duarte, W. F., \& Duarte, J. L. M. (1999). Manual: matrizes progressivas coloridas de Raven. São Paulo: Centro Editor de Testes e Pesquisas em Psicologia.

Aquino, S. M. C. (1999). Promoção cognitiva em estudantes de uma escola agrotécnica: relato de uma experiência. Dissertação de mestrado não-publicada, Universidade Gama Filho, Rio de Janeiro.

Ayoub, C., O'Connor, E., Rappolt-Schlictmannc, G., Vallotond, C., Raikese, H., \& Chazan-Cohenf, R. (2009). Cognitive skill performance among young children living in poverty: risk, change, and the promotive effects of Early Head Start. Early Childhood Research Quarterly, 24 (3), 289-305.

Bransford, J. D., \& Stein, B. S. (1993). The ideal problem solver: a guide for improving thinking, learning, and creativity. New York: W. H. Freeman.

Campbell, F. A., \& Ramey, C. T. (1994). Effects of early intervention on intellectual and academic achievement: a follow up study of children from low income families. Child Development, 65 (2), 684-698.

Carroll, J. B. (1993). Human cognitive abilities: a survey of factor-analytic studies. New York: Cambridge University Press.
Cattell, R. B. (1971). Abilities: their structure, growth, and action. Boston: Houghton Mifflin.

Cattell, R. B. (1987). Intelligence: its structure, growth and action. Amsterdam: Elsevier.

Chariglione, I. P. F. (2010). A influência de diferentes tipos de treinos cognitivos na memória de idosos institucionalizados. Dissertação de mestrado não-publicada, Universidade de Brasília.

Cole, J. C., \& Randall, M. K. (2003). Comparing the cognitive ability models of Spearman, Horn and Cattell, and Carroll. Journal of Psychoeducational Assessment, 21 (2), 160-179.

Colom, R. (2002). Se puede mejorar la inteligencia? En los límites de la inteligencia: es el ingrediente del éxito en la vida? (pp.251-278). Madrid: Ediciones Pirámide.

Colom, R. (2006). O que é inteligência? In C. Flores-Mendoza \& R. Colom (Orgs.), Introdução à psicologia das diferenças individuais (pp.59-72). Porto Alegre: ArtMed.

Colom, R., \& Flores-Mendoza, C. E. (2006). Armazenamento de curto prazo e velocidade de processamento explicam a relação entre memória de trabalho e o fator $g$ da inteligência. Psicologia:Teoria e Pesquisa, 22 (1), 113-122.

Detterman, D. K., \& Sternberg, R. J. (1982). How and how much can intelligence be increased? Norwood: Lawrence Erlbaum.

Flynn, J. R. (2006). O efeito Flynn: repensando a inteligência e aquilo que a afeta. In C. Flores-Mendoza \& R. Colom (Orgs.), Introdução à psicologia das diferenças individuais (pp.387-411). Porto Alegre: Artmed.

Gomes, C. M. A. (2002). Feuerstein e a construção mediada do conhecimento. Porto Alegre: Artmed.

Grotzer, T. A., \& Perkins, D. A. (2000). Teaching of intelligence: a performance conception. In R. J. Sternberg (Org.), Handbook of intelligence (pp.492-515). New York: Cambridge University Press.

Horn, J. L. (1985). Remodeling old theories of intelligence: Gf-Gc theory. In B. B. Wolman (Org.), Handbook of intelligence (pp.267-300). New York: Wiley.

Horn, J. L. (1991). Measurement of intellectual capabilities: a review of theory. In K. S. McGrew, J. K. Werder \& R. W. Woodcock (Orgs.), Woodcock-Johnson technical manual (pp.197-232). Chicago: Riverside.

Irigaray, T. Q. (2009). Efeitos de um treino de atenção, memória e funções executivas na cognição, na qualidade de vida e no bem-estar psicológico de idosos saudáveis. Tese de doutorado não-publicada, Pontifícia Universidade Católica do Rio Grande do Sul, Porto Alegre.

Lynn, R. (2009). Fluid intelligence but not vocabulary has increased in Britain, 1979-2008. Intelligence, 37 (3), 249-255.

Lynn, R., \& Harvey, J. (2008). The decline of the world's IQ. Intelligence, 36 (2), 112-120.

Mayer, R. (2000). Intelligence and education. In R. J. Sternberg (Org.), Handbook of intelligence (pp.519-533). New York: Cambridge University Press.

Melo, M. G. (2007). Tradução e adaptação do subteste de vocabulário do Stanford-Binet-IV para a população brasileira. 
Tese de doutorado não-publicada, Universidade de São Paulo.

Muragaki, C. S., Okamoto, K. H., Furlan, L., \& Toldrá, R. C. (2006). A utilização de jogos pela terapia ocupacional: contribuição para a reabilitação cognitiva. Anais do $X$ Encontro Latino Americano de Iniciação Científica e VI Encontro Latino Americano dePós-Graduação-Universidade do Vale da Paraíba (pp. 2554-2527). São José dos Campos: Univap.

Oliveira-Castro, J. M., \& Oliveira-Castro, K. M. (2001). Função adverbial de "inteligência": definições e usos em psicologia. Psicologia: Teoria e Pesquisa, 17 (3), 257-264.

Perkins, D. N., \& Grotzer, T. A. (1997). Teaching intelligence. American Psychologist, 52 (10), 1125-1133.

Primi, R. (2002). Inteligência fluida: definição fatorial, cognitiva e neurospicológica. Paidéia, 12 (23), 57-75.

Rabbitt, P., \& Stuart-Hamilton, I. (1997). Age-related decline in spelling sbility: a link with fluid intelligence? Educational Gerontology, 23 (5), 437-441.

Schelini, P. W. (2002). Bateria multidimensional de inteligência infantil: proposta de instrumento (pp.1-31). Tese de douto- rado não-publicada, Pontifícia Universidade Católica de Campinas.

Schelini, P. W. (2006). Teoria das inteligências fluida e cristalizada: início e evolução. Estudos de Psicologia, 11 (3), 323-332.

Schelini, P. W., \& Wechsler, S. (2005). Bateria multidimensional de inteligência infantil: desenvolvimento de instrumento. Psico-USF, 10 (2), 129-139.

Stein, L. M. (1994). TDE: Teste de Desempenho Escolar: manual para aplicação e interpretação. São Paulo: Casa do Psicólogo.

Wechsler, D. (2002). WISC-III: Escala de Inteligência Wechsler para Crianças: manual, adaptação e padronização brasileira. São Paulo: Casa do Psicólogo.

Woodcock, R. W. (1998). Extending Gf-Gc theory into practice. In J. J. McArdle \& R. W. Woodcock (Eds.), Human cognitive abilities in theory and practice (pp.137-156). Londres: Lawrence Erlbaum Associates.

Recebido em: 26/11/2010

Versão final reapresentada em: 7/11/2011

Aprovado em: 5/12/2011 